Provided for non-commercial research and education use. Not for reproduction, distribution or commercial use.

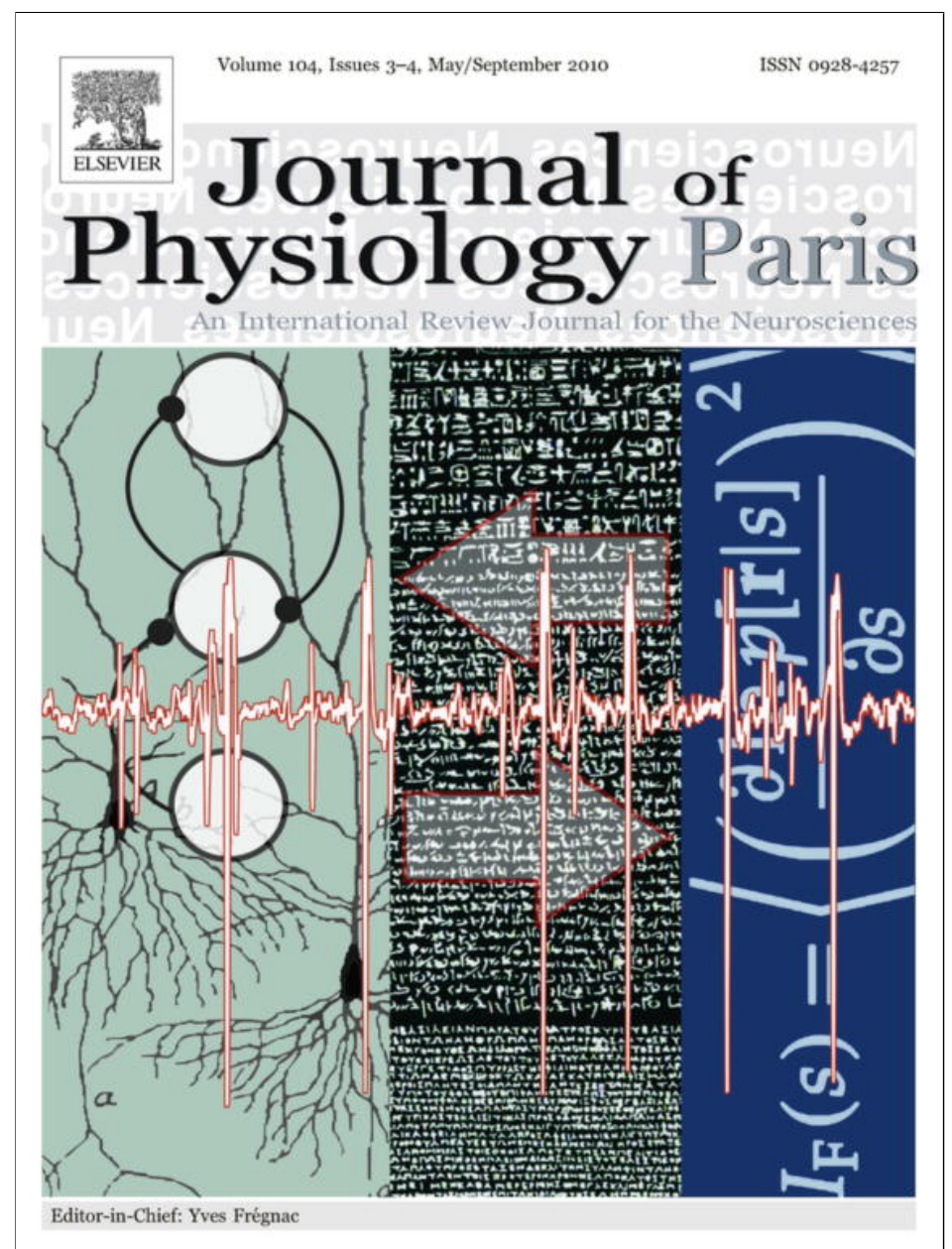

This article appeared in a journal published by Elsevier. The attached copy is furnished to the author for internal non-commercial research and education use, including for instruction at the authors institution and sharing with colleagues.

Other uses, including reproduction and distribution, or selling or licensing copies, or posting to personal, institutional or third party websites are prohibited.

In most cases authors are permitted to post their version of the article (e.g. in Word or Tex form) to their personal website or institutional repository. Authors requiring further information regarding Elsevier's archiving and manuscript policies are encouraged to visit:

http://www.elsevier.com/copyright 


\title{
A biophysical model for modulation frequency encoding in the cochlear nucleus
}

\author{
Manuel C. Eguia ${ }^{\mathrm{a}, *}$, Guadalupe C. Garcia ${ }^{\mathrm{a}}$, Sebastian A. Romano ${ }^{\mathrm{b}}$ \\ ${ }^{a}$ Universidad Nacional de Quilmes, Laboratorio de Acústica y Percepción Sonora, RS Peña 352, Bernal B1876BXD Buenos Aires, Argentina \\ ${ }^{\mathrm{b}}$ Universidade Federal do Rio de Janeiro, Instituto de Biofísica Carlos Chagas Filho, Centro de Ciências da Saúde, bloco G, G2-019 Cidade Universitária, 21941-902 Rio de Janeiro, Brazil
}

\section{A R T I C L E I N F O}

\section{Keywords:}

Computational neuroscience

Auditory system

Cochlear nucleus

Temporal coding

\begin{abstract}
A B S T R A C T
Encoding of amplitude modulated (AM) acoustical signals is one of the most compelling tasks for the mammalian auditory system: environmental sounds, after being filtered and transduced by the cochlea, become narrowband AM signals. Despite much experimental work dedicated to the comprehension of auditory system extraction and encoding of AM information, the neural mechanisms underlying this remarkable feature are far from being understood (Joris et al., 2004). One of the most accepted theories for this processing is the existence of a periodotopic organization (based on temporal information) across the more studied tonotopic axis (Frisina et al., 1990b).

In this work, we will review some recent advances in the study of the mechanisms involved in neural processing of AM sounds, and propose an integrated model that runs from the external ear, through the cochlea and the auditory nerve, up to a sub-circuit of the cochlear nucleus (the first processing unit in the central auditory system). We will show that varying the amount of inhibition in our model we can obtain a range of best modulation frequencies (BMF) in some principal cells of the cochlear nucleus. This could be a basis for a, synchronicity based, low-level periodotopic organization.
\end{abstract}

(c) 2009 Elsevier Ltd. All rights reserved.

\section{Introduction}

The representations of natural sounds in the auditory nervous system are based on dense temporal and population codes in the first stages of processing, and sparse rate and population codes in more central areas. Low level representations are highly redundant and rely on fast and accurate synapses. Higher representations of natural stimuli, in turn, are more specific and in general require less specialized units (Eggermont, 2001). This process of going from more literal to more abstract representations is of great interest in computational neuroscience, since it is closely related to how many different percepts are supposed to emerge from a continuous stimulus in our brains .

Also, natural sounds have broad band spectra, are highly structured in time and formed by segments with durations of a few seconds. Their temporal representation is based both on phase locking to neuronal firing to the fine structure (carrier frequency) and locking of some neurons to the, slower, amplitude modulations (envelope). The encoding of the envelope is one of the main cues used to extract useful percepts from speech signals. This temporal picture coexist with the, more widely studied, tonotopic organization of all the auditory nuclei.

While amplitude modulations are encoded in the auditory nerve using solely synchronized spiking (Joris and Yin, 1992), several response measures on neurons of the ventral cochlear nucleus

\footnotetext{
* Corresponding author.

E-mail address: meguia@unq.edu.ar (M.C. Eguia).
}

have shown enhanced synchronization for a small range of modulation periods only (Rhode and Greenberg, 1994; Frisina et al., 1990a). Therefore, an extra periodotopic (based on temporal information) dimension exists in this neural population, aside of the tonotopic organization inherited from the cochlea (Langner, 1992). Furthermore, in subsequent stages of processing (central nucleus of the inferior colliculus, upstream in the auditory pathway) the periodotopic information is also expressed in rate response (Schreiner and Langner, 1988; Krishna and Semple, 2000). For a review see Frisina (2001) and Joris et al. (2004).

Physiological responses to amplitude-modulated (AM) stimuli have provided a basic description of how controlled fluctuations in a signals temporal envelope are represented at several levels of the auditory pathway. The same class of stimulus has also been used extensively in psychophysical experiments, as a convenient means to investigate perceptual temporal processing capabilities and limitations (Fassel and Kohlrausch, 1995; Fastl and Zwicker, 2007).

In fact, most natural sounds are modulated in amplitude. After being processed by the cochlea, the acoustical signal is decomposed in many signals that run in parallel channels throughout the auditory nerve (AN) with a narrowband representation. Each of these channels conveys a signal that is limited in frequency (around their characteristic frequency, CF), but strongly modulated. At the end of the processing by the cochlea, the information about the stimulus is provided by: (1) a measure of the activity of each channel, (2) the characteristic frequency of the channel (fine structure) that is encoded in the phase locking of the AN, and (3) 
the envelope modulation that provide temporal information at a longer time scale (relative to the $\mathrm{CF}$ ). Many natural stimuli, such as human speech, are nearly stable in the representations provided by (1) and (2), but strongly encoded by (3).

Several models for the processing and encoding of amplitude modulated sounds were proposed so far (Hewitt et al., 1991; Hewitt and Meddis, 1994; Eriksson and Robert, 1999; Nelson and Carney, 2004; Borst et al., 2004; Guérin et al., 2006; Dicke et al., 2006; Bahmer et al., 2006). Some of these works were mainly centered in the replication of the temporal responses to AM stimuli, but others were also aimed at obtaining a sort of periodotopic organization in the system. This means that there are subpopulations tuned to certain modulation frequencies of the stimulus, across the tonotopic axis. In the cochlear nucleus, this tuning was observed more in terms of preferential synchronization rather than rate enhancing.

Even when these models succeeded in obtaining the periodotopic structure, some of them are too abstract or with details that could not be implemented biologically. To our knowledge, at least two recent contributions are the exception. Dicke and collaborators proposed a simple midbrain network that is able to encode the modulation frequencies as a place-rate code at the level of the inferior colliculus (Dicke et al., 2006). However they were less interested on the synchronization coding at the level of the cochlear nucleus. In the second case, Bahmer and Langner proposed a biologically feasible neural network for the cochlear nucleus that is accurate both in terms of modulation encoding and dynamical range (Bahmer et al., 2006). But, until now, there was no evidence of a selective tuning mechanism for modulation frequencies in this model.

In this work, we propose a biologically rooted, dynamically simplified, and hopefully accurate model of modulation frequency encoding in the cochlear nucleus that is able to reproduce the observed synchronization tuning of $\mathrm{CN}$ cells. We combine a highly detailed implementation of the auditory periphery with realistic, channel-based, neuron models and a simplified and flexible network structure.

All parameters in our model retain a clear biological significance, and most of them are also constrained by experimental observations in the auditory system of mammals. A few model parameters are related to neural processes that are either elusive to experimental quantification or very controversial, with contradictory results reported in the literature. In this last case, the parameters will be explored within the range of physiologically plausible values.

\section{Computational model}

In the mammalian auditory system, the acoustical information is first encoded into the auditory nerve by the cochlea. Then, the auditory nerve fibers terminate in the first processing stage of the auditory system: the cochlear nucleus, a complex formed by a large variety of neurons that extract specific features from the signal representation observed at the level of the auditory nerve.

Our model is composed by two main stages: (a) auditory periphery and (b) a small network belonging to cochlear nucleus (CN). The first one comprises the external and middle ear, the cochlea and the auditory nerve. Its output are the spike trains conveyed by the auditory fibers. The second part is composed by three different types of $\mathrm{CN}$ neurons, their connections and their synapses with the AN.

\subsection{Auditory periphery}

The auditory periphery model is based both on previously proposed models (Shamma et al., 1986; Nobili et al., 1998; Meddis,

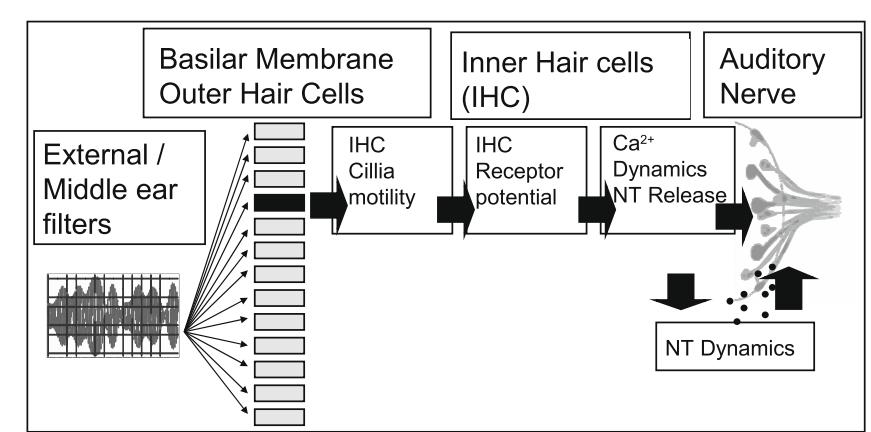

Fig. 1. Schematic diagram of the complete composite auditory peripheral model. It is composed of: (1) basilar membrane (BM), including outer hair cells (OHC); (2) inner hair cells (IHC): cilia motility, receptor potential, calcium and neurotransmitter dynamics; and (3) auditory nerve (AN). The discharge probability produced by the IHC is transformed into spike trains at the auditory nerve level.

1986; Zhang and Carney, 2005; Sumner et al., 2002) and new research that is not contained in those works (Sikora et al., 2005; Rodríguez-Contreras and Yamoah, 2003; Goutman and Glowatzki, 2007). A schematic view is displayed in Fig. 1. There are four main modules: (1) outer/middle ear and basilar membrane, (2) inner hair cells, (3) ribbon synapses, and (4) auditory nerve. After the first module, the signal splits in parallel channels, each corresponding to a different part of the cochlea. At this point these channels are a discretization of the space coordinate, but later will be assigned to a certain number of auditory nerve fibers.

The model is biophysical in spirit, but some processes are simplified as far as their dynamics is not affected and the parameters maintain their biological meaning. Some not yet fully understood phenomena are not included, such as the role of efferent fibers in hair cells and other descending pathways. In this way, the auditory periphery model can be viewed as a unidirectional, modular process with no feedback and without interactions between the parallel channels.

A brief overview of the processes involved in the auditory periphery follows: Sound enters through the external ear and is mechanically filtered, amplified and injected into the cochlea by the ossicles of the middle ear (Dallos, 1996). The interior of the cochlea contains three fluid filled tubes, separated by membranes. The basilar membrane, which forms the partition between two compartments, is a complex structure upon which auditory transduction occurs. Fluctuating pressure difference between the compartments, caused by sound waves, would move the basilar membrane. The organ of Corti resides on top of the basilar membrane and contains the mechano-electrical transducers: the hair cells. It supports three rows of outer hair cells $(\mathrm{OHC})$ and one row of inner hair cells (IHC) (Dallos, 1996). These cells play different roles in transduction: IHC act as the primary receptor cell for the auditory system and the OHC can also act as motor cells, increasing hearing sensitivity and frequency selectivity (Choe et al., 1998). The movement of the basilar membrane displaces hair bundles attached to the IHC, causing a depolarization of the cell, which in turn results in a receptor potential. Hair cells are also presynaptic terminals, the basolateral membrane of each cell contains several presynaptic actives zones, where chemical neurotransmitter is released. In mammals, 5-30 auditory fibers contacts one inner hair cell trough a single unbranched dendrite, in a single ribbon synapses (Moser et al., 2006). The probability of a spike occurring in a fiber is directly proportional to the concentration of neurotransmitter in the synaptic cleft (Carney, 1993).

\subsubsection{Outer-middle ear and basilar membrane}

We implemented a detailed biophysical model of the basilar membrane and cochlear hydrodynamics, based on the original 
work of Nobili and collaborators (Nobili et al., 1998). This model considers the basilar membrane as a set of oscillators with mass, stiffness and viscosity dependent on the belonging site of the basilar membrane, subject to external forces generated by the acceleration of the stapes, and transmitted by the cochlear fluid and the outer hair cells. The presence and interaction of the tectorial membrane with the $\mathrm{OHC}$ is included as a second set of damped oscillators, coupled to the movement of OHC cilias. Therefore, the outer hair cell force terms, in the linear approximation, behave like negative viscosity terms and undamped cochlear motion, altering the properties of the basilar membrane.

A complete description of the model equations can be found in Nobili et al. (1998). Parameters were adjusted to values corresponding to the human cochlea (Nobili et al., 2003). The outer and middle ear were modeled as a two-pole band-pass filter (Shamma et al., 1986).

\subsubsection{Inner hair cells}

Deflection of the stereocilia bundles modulates the flow of ions into the cells and thus causes fluctuations of their intracellular potential. The following simple model for the IHC was proposed by Shamma and collaborators (Shamma et al., 1986). It assumes that IHC receptor potential is primarily controlled by the interplay of a transducer inward $\mathrm{K}^{+}$current that results from stereocilia deflections and a outward $\mathrm{K}^{+}$basolateral current, that eliminate the excess of $K^{+}$from within the IHC. The proposed electric equivalent circuit for the cell is composed by several components that reflect the electrical properties of the cell and the surrounding fluid. The membrane potential of the cell body is modeled with a passive electrical analog circuit. The stereocilia movements cause a change in the number of open ion channels, and thus in the apical conductance. They are driven by the fluid motion, so that cilia moves in phase with basilar membrane velocity at low frequencies and with displacement at high.

A complete description of the IHC model equations and parameters values can be found in Shamma et al. (1986). We choose this model because is the simplest one that retains the biological meaning of the parameters and is accurate enough, at least for intensities between 30 and $80 \mathrm{~dB}$ and for quasi-stationary stimuli. Additional conductances could be needed if we move out from these conditions, see LopezPoveda and Martin (2006)

\subsubsection{Ribbon synapses}

The release of neurotransmitter in the synaptic cleft is mediated by calcium ions in all synapses. The dynamics of transmitter release is the most critical part for the signal transduction in the auditory periphery. Then we model the dynamics in detail as a three part process: (1) activation of calcium ion channels, (2) calcium dynamics and binding to vesicles, and (3) neurotransmitter dynamics.

We employ a first order process to model the calcium current density $\left(I_{C a}\right)$, for recently observations (Goutman and Glowatzki, 2007), with a time constant that is not voltage dependent. Thus, the current is a function of the cell membrane potential $V$ :

$I_{C a}(t)=G_{C a}^{m a x} m_{I_{C a}}(t)\left(V(t)-E_{C a}\right)$

where $E_{C a}$ is the reversal potential for calcium and $G_{C a}^{\max }$ is the calcium conductance density (in units of $S / \mathrm{cm}^{2}$ ) in the vicinity of the synapse, with all the channels open. Since conductance density is expressed in $\mathrm{S} / \mathrm{cm}^{2}$ and potentials in $\mathrm{mV}$, the volumetric calcium current has units of $\mathrm{mA} / \mathrm{cm}^{2}$. The variable $m_{I_{C a}}$ is the fraction of calcium channels that are open and depends on the membrane potential. The steady-state value of the latter, $m_{I_{c a} \infty}$ is modeled by a Boltzmann function (Sumner et al., 2002)
$m_{I_{C a}, \infty}=\left[1+\beta_{C a}^{-1} \exp \left(-\gamma_{C a}\right) V(t)\right]^{-1}$

where $\beta_{C a}$ and $\gamma_{C a}$ are constants chosen to reflect published observations of calcium currents, and $m_{I_{C a}}$ is a low-pass-filtered function of $m_{I_{C a}, \infty}$ (Rodríguez-Contreras and Yamoah, 2003).

$\tau_{C a} \frac{d m_{I_{C a}}}{d t}+m_{I_{C a}}=m_{I_{C a}, \infty}$

The calcium concentration is modeled as a first-order low-passfiltered function of calcium current (Hudspeth and Lewis, 1988).

$\frac{d\left[\mathrm{Ca}^{+2}\right]_{d}}{d t}(t)=-\frac{I_{C a}}{2 F d}-\frac{\left[\mathrm{Ca}^{+2}\right]_{d}}{\tau_{C a}}$

where $\left[\mathrm{Ca}^{+2}\right]_{d}$ is the calcium concentration (in units of mMol) at depth $d$ (in $\mathrm{mu}$ ) inside the membrane, $F$ the Faraday $(96,489 \mathrm{C} /$ Mol) constant and $\tau_{\mathrm{Ca}}$ the time constant of removal for calcium. The factor 2 is the valence of calcium ions (Sikora et al., 2005).

The relationship between calcium concentration and the release of vesicles is a critical factor in determining the behavior of the model. The form of this dependence is still a matter of debate. While many researchers found a super-linear dependence of release on $\mathrm{Ca}$, indicating cooperative behavior, more recent studies show that for physiological relevant voltage range and concentration values hair cell synapses operate with a linear $\mathrm{Ca}$ dependence of release (Hudspeth and Lewis, 1988; Goutman and Glowatzki, 2007). Based on this, we only assume a saturating function under the form of a Hill equation with a linear exponent.

$k=\frac{k_{\max }\left[\mathrm{Ca}^{+2}\right]_{d}}{A+\left[\mathrm{Ca}^{+2}\right]_{d}}$

where $k_{\max }$ is the maximum release rate and $A$ is an adjustable parameter (Sikora et al., 2005).

The last part of the IHC model contains the dynamics of the neurotransmitter and accounts for the IHC-AN adaptation observed in all fibers. We use a two-variable model proposed by Zhang and Carney (2005), as a dynamical reduction of a model proposed by Meddis and collaborators (Meddis, 1986). The neurotransmitter dynamics can be described by the following equations:

$$
\begin{aligned}
& \frac{d q}{d t}=y(M-q(t))+x w(t)-k(t) q(t) \\
& \frac{d w}{d t}=k(t) u q(t)+x w(t)
\end{aligned}
$$

where $q$ is the concentration of free neurotransmitter and $w$ the concentration of neurotransmitter in a reprocessing store. New free transmitter is either manufactured from the factory at a rate $y(M-q)$, where $M$ is the amount of neurotransmitter in a global store (ribbon synapses), provided by the reprocessing store at a rate $x w$, or released to the synaptic cleft at a rate $k q$. The transmitter in the cleft is reprocessed at a rate kuq. All parameters for this model stage were adjusted in order to obtain an accurate auditory nerve representation, and are listed in Table 1.

\subsubsection{Auditory nerve}

The final stage of the peripheral model converts the output of the synapse into discharge times, providing a time varying input $(k q)$ to a Poisson discharge generator. The occurrence of a discharge influences the probability of pursuant discharges through absolute and relative refractory effects. Discharges are not allowed to occur during the absolute refractory time interval, and there is an exponentially decreasing inhibition of the probability of firing after that refractory time. The model equations and parameters are the same as in Carney (1993).

Three types of fibers can be distinguished from their spontaneous discharge rates and dynamic range: low threshold high-spon- 
Table 1

Parameters values for the auditory periphery.

\begin{tabular}{|c|c|c|}
\hline Parameter & Typical value & Description \\
\hline \multicolumn{3}{|l|}{$\begin{array}{l}\text { Calcium } \\
\text { dynamics }\end{array}$} \\
\hline$\tau_{m}$ & $0.1 \mathrm{~ms}$ & Calcium current time constant \\
\hline$E_{C a}$ & $66 \mathrm{mV}$ & Calcium reversal potential \\
\hline$G_{C a}^{\max }$ & {$\left[\begin{array}{lll}0.003 & 0.01 & 0.03\end{array}\right] \mathrm{s} / \mathrm{cm}^{2}$} & Maximal calcium conductance \\
\hline$\tau_{C a}$ & $0.1 \mathrm{~ms}$ & $\begin{array}{l}\text { Time constant of removal for } \\
\text { calcium }\end{array}$ \\
\hline$\gamma_{c a}$ & $0.6 \mathrm{mV}^{-1}$ & $\begin{array}{l}\text { Boltzmann's function } \\
\text { parameters }\end{array}$ \\
\hline$\beta_{C a}$ & 10,000 & $\begin{array}{l}\text { Boltzmann's function } \\
\text { parameter }\end{array}$ \\
\hline$k_{\max }$ & $0.4 \mathrm{~ms}^{-1}$ & $\begin{array}{l}\text { Maximum release rate of } \\
\text { neurotransmitter }\end{array}$ \\
\hline$A$ & $10 \mu \mathrm{M}^{-1}$ & Parameter of the Hill equation \\
\hline$F$ & $96,489 \mathrm{C} / \mathrm{Mol}$ & The Faraday constant \\
\hline$d$ & $0.1 \mu \mathrm{m}$ & $\begin{array}{l}\text { Depth inside the presynaptic } \\
\text { membrane }\end{array}$ \\
\hline \multicolumn{3}{|l|}{ Synapse } \\
\hline Y & {$\left[\begin{array}{llll}0.010 & 0.00948 & 0.0103\end{array}\right] \mathrm{ms}^{-1}$} & Replenishment rate \\
\hline$X$ & {$\left[\begin{array}{llll}0.140 & 0.149 & 0.150\end{array} \mathrm{~ms}^{-1}\right.$} & Reprocessing rate \\
\hline$U$ & {$\left[\begin{array}{llll}0.86 & 0.87 & 0.87\end{array}\right]$} & A dimensional parameter \\
\hline$M$ & {$\left[\begin{array}{lll}8.6 & 8.5 & 8.6\end{array}\right]$} & $\begin{array}{l}\text { Maximum amount of free } \\
\text { transmitter }\end{array}$ \\
\hline
\end{tabular}

taneous-rate (HSR), medium-spontaneous-rate (MSR), and high threshold low-spontaneous-rate (LSR) (Johnson, 1980; Liberman, 1978). These different neuronal responses are originated at the synapses varying the maximal conductance of calcium $G_{C a}^{\max }$ (Sumner et al., 2002). We assign 100 fibers to each channel, since this corresponds to $\mathrm{a} \approx 100 \mu$ partition of the cochlea.

\subsection{Cochlear nucleus}

All information from the auditory periphery is carried via AN fibers to the cochlear nucleus $(\mathrm{CN})$. This complex is divided, on the basis of differing cytoarchitecture (Osen, 1969) and the existence of three separate tonotopic frequency maps (Rose et al., 1960), into three major divisions: anteroventral (AVCN), posteroventral (PVCN), and dorsal (DCN). These three divisions also show broadly different physiological response properties, and it is likely, therefore, that they each play a different functional role in the perception of sound.

In contrary to the auditory nerve whose response is monotype (primary response), there are several different responses observed in the cochlear nucleus. These responses are related to a collection of neural circuits which form the $\mathrm{CN}$, and which are diverse both in anatomical and physiological terms. These vary from the simplest system, the bushy cell in the ventral cochlear nucleus with primary-like response, to more complex responses located in the dorsal cochlear nucleus. It is generally understood that these responses convey information about a particular aspect of the stimulus and that at the $\mathrm{CN}$ the monotype response of the auditory nerve is branched in specialized parallel pathways (Young and Oertel, 2004).

\subsubsection{Cell units}

We will focus on one of these possible pathways, specialized in the encoding of amplitude modulated signals. Pioneering studies of AM responses in the cochlear nucleus were done by Møller (1974). Even when the majority of principal cells in the $\mathrm{CN}$ have been found to synchronize to modulation frequency better than AN fibers, stellate cells in the AVCN and PVCN have very high synchronization rates and, most interestingly, in some cases display bandpass time modulation transfer functions (Joris et al., 2004). This last feature can be assimilated to a modulation frequency tuning (Rhode and Greenberg, 1994; Frisina et al., 1990a).

Stellate neurons (also called multipolar) have been divided into two subtypes that can be distinguished by their projections, morphology and their response to stationary stimuli (Palmer et al., 2003; Paolini et al., 2005). The most numerous group is formed by T-stellate cells that project to the trapezoidal body, are aligned with the isofrequency layer of the tonotopic map (hence they are excited in a very narrow characteristic frequency), and respond with a chopping behavior maintained throughout the stimulus. T-stellate cells are referred also as choppers, based in their poststimulus responses and are glutamatergic (Doucet and Ryugo, 2006). The other group is formed by D-stellate cells that project to the DCN, have a dendritic span covering a wide range of characteristic frequencies (CF), and respond to pure tones and noise with an initial short series of well timed spikes, followed by a decrease of activity. These neurons are also called onset-choppers and are inhibitory and glycinergic. D-stellate cells are an important source of wideband inhibition in the CN (Zhang and Oertel, 1993).

One well-known target of this wideband inhibition are vertical (or tuberculoventral) cells in the DCN. Moreover, this type of neuron sends back inhibitory inputs to the VCN (Wickesberg and Oertel, 1991). Therefore, in our simple circuit it is almost mandatory to include vertical cells of the DCN. Vertical cells respond to stationary inputs with onset, onset-graded or fast chopping patterns (Rhode, 1999), are as narrowband as the T-stellate cells and are glycinergic.

All three type of cells (T-stellate, D-stellate and vertical) are simulated using the model proposed by Rothman and Manis (2003) for VCN neurons. Even when this model was originally adjusted for the VCN, we obtained results very similar to those observed in cat vertical cells (Rhode, 1999), after adjusting the network parameters described in Section 2.2.3.

This model consists of a single electrical compartment with a membrane capacitance, a fast inactivating sodium current, a high threshold potassium current, a hyperpolarization-activated (inward cationic) current and a leakage current. All model equations are the same as described in the appendix of Rothman and Manis (2003).

The input for these units is provided by a synaptic current $I_{s y n}(t)$ arriving at the soma, which is the sum of: (a) glutamatergic inputs (dendritic or somatic) of the synapses with auditory nerve fibers for all cells, (b) glutamatergic (excitatory) somatic inputs originated in the T-stellate cells, and (c) glycinergic (inhibitory) somatic inputs originated both in D-stellate and vertical cells. Of course, the last two currents are only present when there is an experimentally validated connection between two $\mathrm{CN}$ cells (see Section 2.2.3).

\subsubsection{Innervation of $\mathrm{CN}$ cells}

The three types of cells receive direct input from the auditory nerve and using the same type of contact (button endings) with slightly different time constants (Ryugo and Parks, 2003). The main difference reside in the $C F$ span: $D$-stellate neurons receive inputs from a wide cochlear region (CF span ranging between 1 and 1.5 octaves), while T-stellate and vertical cells are aligned with nerve fibers and narrowband. Also cells differ in the number and site of contacts. Both vertical and T-stellate receive very few (5-10) dendritic inputs. D-stellate cells receive numerous $(\approx 100)$ inputs on their dendritic trees, but also integrate strong somatic inputs, only from fibers coming from the center $\mathrm{CF}$.

The number, type, site of contact, and CF span of the auditory fibers arriving to each cell in our model is the following. For T-stellate and vertical cells there are 10 AN fibers (4 HSR, 3 MSR and 3 LSR) coming from a single channel of the auditory periphery model (the central CF) and their inputs are always filtered by the dendrite. For D-stellate there are $10 \mathrm{AN}$ fibers with the same type distribu- 
tion for somatic inputs coming from the central CF and 4 AN fibers (2 HSR, 1 MSR and 1 LSR) coming from each of the 20 nearest channels and making contact with the dendritic arborization.

We modeled the synapses in the button endings using a doubleexponential alpha function, with parameters adjusted in order to reproduce the results obtained in Gardner et al. (1999) for stellate and vertical post-synaptic currents.

$$
\alpha(t)=C_{0} \frac{t}{\tau_{0}} \exp \left[1-\frac{t}{\tau_{0}}\right]+C_{1} \frac{t+t_{1}}{\tau_{1}} \exp \left[1-\frac{t+t_{1}}{\tau_{1}}\right]
$$

for $t>0$ and $\alpha(t<0)=0$. Parameters for both type of synapses (stellate and vertical) are given in Table 2. The normalized spike train of each fiber converging to the soma or neuropil is convolved with Eq. (8), and filtered with a dendrite low-pass filter if it contacts the neuropil. Dendritic filtering was simulated as a first-order lowpass filter with $6 \mathrm{~dB}$ attenuation per octave and a cutoff frequency of $300 \mathrm{~Hz}$.

\subsubsection{CN network}

During the last decade, a major advance in elucidating the neuronal circuitry of the CN was made (Ferragamo et al., 1998; Wickesberg and Oertel, 1991; Arnott et al., 2004; Needham and Paolini, 2006; Young and Oertel, 2004). Ultrastructuctural, tracer, neurotransmitter release, and antidromic studies have provided a considerable amount of evidence supporting that: (a) D-stellate cells inhibit T-stellate neurons, ipsi and contra-laterally (Ferragamo et al., 1998); (b) T-stellate cells form mutually excitatory circuits (Ferragamo et al., 1998; Bahmer et al., 2006); (c) D-stellate cells are the source of wideband inhibition in the DCN and vertical cells are among their targets (Arnott et al., 2004; Doucet et al., 1999); and (d) vertical cells are a source of narrowband inhibition in the VCN, making contacts with stellate cells (Wickesberg and Oertel, 1991). Hence, a recurrent network exists between T-stellate and D-stellate in the VCN and vertical cells in the DCN, all of which are driven by the auditory nerve. There are other, less well established connections, that involve small cells in the $\mathrm{CN}$ and olivocochlear efferents.

We propose a minimalist circuit formed by four units: two mutually excitatory T-stellate (T1 and T2) neurons, one D-stellate neuron (D) and one vertical cell (V). D-stellate and vertical units

Table 2

Fixed parameters values for the cochlear nucleus.

\begin{tabular}{lll}
\hline Parameter & $\begin{array}{l}\text { Value } \\
(\mathrm{ms})\end{array}$ & Description \\
\hline $\begin{array}{c}\text { Synapse AN - } \\
\text { stellate cells }\end{array}$ & & \\
$C_{0}^{S}$ & 0.8 & Coefficient for fast exponential \\
$\tau_{0}^{S}$ & 0.1 & Fast decaying time constant \\
$C_{1}^{S}$ & 0.2 & Coefficient for slow exponential \\
$\tau_{1}^{S}$ & 0.5 & Slow decaying time constant \\
$t_{1}^{S}$ & 0.5 & Time offset for slow exponential \\
Synapse AN - & & \\
\multicolumn{1}{c}{ vertical cells } & & \\
$C_{0}^{V}$ & 0.8 & Coefficient for fast exponential \\
$\tau_{0}^{V}$ & 0.19 & Fast decaying time constant \\
$C_{1}^{V}$ & 0.2 & Coefficient for slow exponential \\
$\tau_{1}^{V}$ & 0.9 & Slow decaying time constant \\
$t_{1}^{V}$ & 0.54 & Time offset for slow exponential \\
Synaptic delays & & \\
$\tau_{T T}$ & 0.4 & Synaptic delay between T-stellate cells \\
$\tau_{D T}$ & 0.2 & Synaptic delay between D and T-stellate cells \\
$\tau_{V T}$ & 2 & Synaptic delay between vertical and T-stellate cells \\
$\tau_{V D}$ & 2 & Synaptic delay between vertical and D-stellate cells \\
$\tau_{D V}$ & 2 & Synaptic delay between D-stellate and vertical cells \\
\hline
\end{tabular}

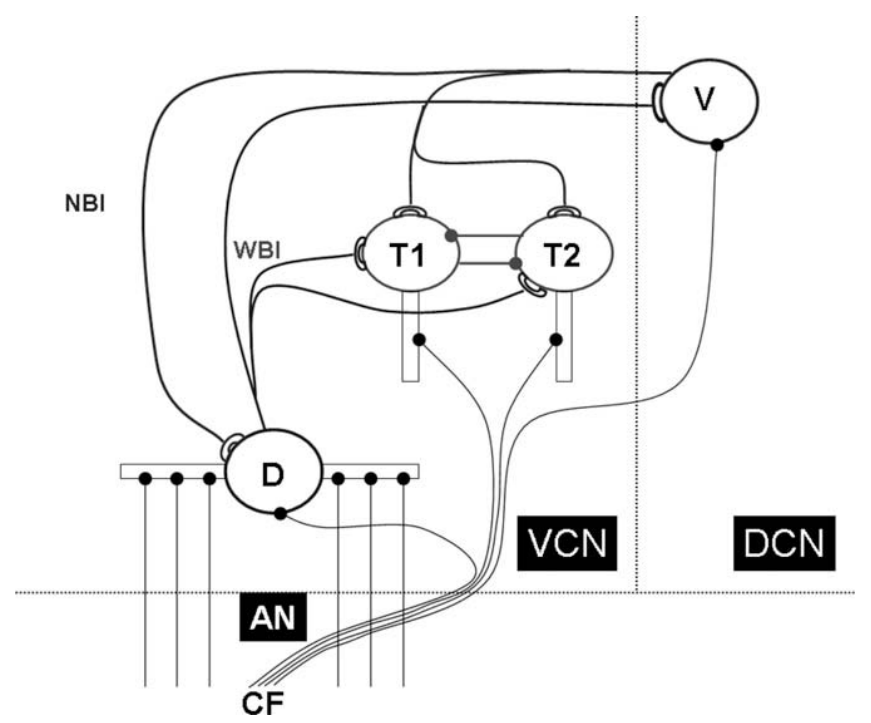

Fig. 2. Proposed network model. Auditory nerve fibers (AN) innervate a small network of four cells for a given characteristic frequency (CF). The units are: (D) Dstellate neuron, glycinergic, receives input form a wide range of $\mathrm{CF}$ and acts as a wideband inhibitor (WBI) over the $\mathrm{CN}$; (T1) and (T2) two mutually excitatory Tstellate neurons; and (V) vertical cell of the dorsal cochlear nucleus (DCN), glycinergic, and acts as a narrowband inhibition (NBI) on the ventral cochlear nucleus (VCN).

make inhibitory projections to the other three cells. The schematic drawing of this small network is displayed in Fig. 2. The innervation afferent pattern was explained in the previous section, and there are no lateral (across $\mathrm{CF}$ ) connections at this point.

The synapses between cells are modeled using the same alpha function described before in Eq. (8) for glycinergic and glutamatergic inputs, but a short time delay is also introduced in order to take into account the experimental observation of delay between pulses in monosynaptic and polisynaptic IPSP (Ferragamo et al., 1998). The synaptic delays are listed in Table 2.

The strengths of the synaptic connections are the main parameters to be varied in our model. In this way, we have a fixed physiological model and a fixed network structure, but the amount of excitation and inhibition is variable. All $\mathrm{CN}$ cells receive a balance of excitatory and inhibitory inputs. Inhibitory inputs are thought to be critical in determining the observed (chopping) behavior and the temporal synchronization in stellate neurons (Paolini et al., 2005).

Now we turn into a detailed account of all the connection parameters. First, we describe the synaptic inputs coming from the auditory fibers referred in the previous section. To be concrete, the final obtained variable, after convolving the normalized spike train coming from the $j$ th auditory fiber of the $i$ th channel with the alpha function (Eq. (8)), will be denoted as $R_{x j}^{i}(t)$, where $x$ is a tag denoting the cell (T1, T2, D, or V for the first T-stellate, the second T-stellate, the D-stellate and the vertical neuron, respectively). This variable corresponds to the fraction of post-synaptic open channels (Destexhe et al., 1999) for a single button ending. The conductances for all the synapses with the auditory nerve can be written as:

$$
\begin{aligned}
& G_{A N}^{T 1}(t)=g_{A T} \sum_{j=1}^{10} R_{T 1 j}^{0}(t) \\
& G_{A N}^{T 2}(t)=g_{A T} \sum_{j=1}^{10} R_{T 2 j}^{0}(t) \\
& G_{A N}^{D n}(t)=g_{A D} \sum_{i=-10}^{10} \sum_{j=1}^{4} R_{D j}^{i}(t)
\end{aligned}
$$


$G_{A N}^{D s}(t)=g_{A D} \sum_{j=1}^{10} R_{D j}^{0}(t)$

$G_{A N}^{V}(t)=g_{A V} \sum_{j=1}^{10} R_{V j}^{0}(t)$

Note that, for D-stellate neurons, we differentiate the total synaptic conductance in the soma $\left(G_{A N}^{D s}\right)$ and in the neuropil $\left(G_{A N}^{D n}\right)$. Also, the indexing of the AN channels starts at the $C F(i=0)$.

The contribution of the AN to the final synaptic currents can be obtained multiplying these conductances by the local membrane potential and performing the dendritic filtering (with the exception of $G_{A N}^{D s}$ ).

The maximum conductances $g_{A x}$ are adjusted in order to obtain a response comparable to experimental data, as detailed in Section 3.2.

The other contribution is formed by the synapses between $\mathrm{CN}$ neurons. Using the same convention as before, the alpha-convoluted normalized spike train coming from unit with label $y$, arriving at neuron with label $x$ is denoted as $R_{y x}$. We write down the total conductances for these synapses.

$G_{g l u}^{T 1}(t)=g_{T T} R_{21}\left(t-\tau_{T}\right)$

$G_{g l u}^{T 2}(t)=g_{T T} R_{12}\left(t-\tau_{T}\right)$

$G_{g l y}^{T 1}(t)=g_{D T} R_{D 1}\left(t-\tau_{D T}\right)+g_{V T} R_{V 1}\left(t-\tau_{V T}\right)$

$G_{g l y}^{T 2}(t)=g_{D T} R_{D 2}\left(t-\tau_{D T}\right)+g_{V T} R_{V 2}\left(t-\tau_{V T}\right)$

$G_{g l y}^{D}(t)=g_{V D} R_{V D}\left(t-\tau_{V D}\right)$

$G_{g l y}^{V}(t)=g_{D V} R_{D V}\left(t-\tau_{D V}\right)$

where the subscript glu denotes excitatory glutamatergic inputs and gly inhibitory glycinergic. There are five fixed synaptic delays $(\tau)$ and five adjustable parameters: the maximum conductances $\left(g_{T T}, g_{D T}, g_{V T}, g_{V D}\right.$, and $\left.g_{D V}\right)$. It is assumed that the coupling between T-stellate cells is symmetric. Note also that we drop the $T$ in subindices in the Rs where there is no ambiguity (ex. T2T1 is written 21 ).

The synaptic currents can be obtained inserting the conductances $G_{i}$ in a current term of the form $G_{i}\left(V-E_{i}\right)$, where $V$ is the local membrane voltage and $E_{i}$ the reversal potential. We use a zero reversal potential for glutamatergic synapses and $E_{g l y}=-80 \mathrm{mV}$ for the glycinergic inputs. All currents were integrated using the multiple synapse algorithm described in Destexhe et al. (1999).

\section{Results}

Having defined our model with the sets of parameters that will be held fixed (see Table 2), and the control parameters, we now examine whether the model can reproduce the observed response of $\mathrm{CN}$ neurons to AM stimuli.

We first define our stimuli and the synchronization measures that will be used, and then analyze two different standard characterizations for the stellate cells: post-stimulus time responses and modulation transfer functions.

\subsection{Stimuli and synchronization measures}

Our stimulus set consists of short (300-500 ms) sinusoidal AM tones, ranging $50-80 \mathrm{~dB}$ SPL, with $100 \%$ modulation depth and a carrier frequency of $1 \mathrm{kHz}$. Modulation frequencies were varied between 10 and $200 \mathrm{~Hz}$.

We choose this type of signal because it is a commonly used stimulus and the range of intensities is the same as the one reported to elicit an enhanced synchronization to modulation in $\mathrm{CN}$ cells (Frisina, 2001). We limited our exploration to only one carry- ing frequency because we are interested on the network parameters that make possible a modulation frequency tuning, or the existence of some type of periodicity organization, aside from the more studied tonotopic one.

The simulation of the response to a single AM tone consumed about $10 \mathrm{~s}$ of CPU time. Therefore, the modulation frequencies and intensity values could be varied almost continuously for a given set of control parameters (maximum conductances for the synapses between $\mathrm{CN}$ cells).

The response of the cell was studied comparing their post-stimulus time histograms (PSTH) with the histograms obtained from experimental observation (Paolini et al., 2005). The PSTH are simply calculated as the histogram of the averaged responses to multiple presentations of the stimulus in identical conditions.

There are different synchronization measures. We adopt here the most widely used metric: the vector strength, also called synchronization index (SI). Each spike is treated as a unit length vector with a phase obtained as the spike time modulo the period of interest. If the time of occurrence of a train of $n$ spikes is contained in the vector $\left\{t_{i}\right\}$, then the synchronization index relative to frequency $f$ can be calculated as:

$\operatorname{SI}(f)=\frac{1}{n} \sqrt{\left(\sum_{i}^{n} \cos \left(2 \pi f t_{i}\right)\right)^{2}+\left(\sum_{i}^{n} \sin \left(2 \pi f t_{i}\right)\right)^{2}}$

In order to detect an enhancement of synchrony we calculate the SI as a function of the modulation frequency, or time modulation transfer function (tMTF).

\subsection{Post-stimulus responses}

We first made a preliminary exploration varying both the amount of inhibition of D-Stellate and vertical cells, and the mutual excitation of T-stellate units, in the range of $0-30 \mathrm{nS}$ for the total maximum synaptic conductances. We determined the existence of a wide region in parameter space where the post-stimulus responses were compatible with that reported in the literature. Making perturbations to the conductance values from there, we detected the more sensitive parameters in terms of their influence on the stimulus response.

The synaptic strength of the mutual excitation of T-stellate cells $\left(g_{T T}\right)$, appears to be the most critical parameter that helps maintain a regular chopping behavior in a wide dynamical range. This is consistent with the results obtained by Bahmer (Bahmer et al., 2006) for a recursive network of 3 and 5 chopper cells. Typical $g_{\text {TT }}$ values that preserve a robust chopping response are in the range 5-10 nS. The amount of wideband inhibition on the chopper unit, governed by $g_{D T}$, only has a marginal influence on the chopper response, at least for sinusoidal AM tones. The wideband inhibition is probably more effective as a way of enhancing the frequency tuning in noisy environments (Pressnitzer et al., 2001) and extracting spectral features from sounds (Rhode and Greenberg, 1994; Paolini et al., 2005).

A typical PSTH for a T-stellate cell (T) in our network during and little after a $250 \mathrm{~ms}$ pure tone stimulus at the characteristic frequency of $1 \mathrm{kHz}$ is displayed in Fig. 3a. The most prominent feature of this type of cells is the chopping behavior in response to tones: a repetitive spiking with regular intervals, unrelated to the stimulus frequency. The discharge rate and the regularity of the time intervals usually decrease after an initial well-timed discharge. This is reflected in the shape of the PSTH (Fig. 3a), which has a very clear pattern at the beginning with peaks at $\approx 4 \mathrm{~ms}$ and barely recognizable peaks at the end.

This pattern changes dramatically when we add modulation to the sinusoidal tone. In Fig. 3b, we display the response of the same unit with the same network parameters, to an AM tone with mod- 

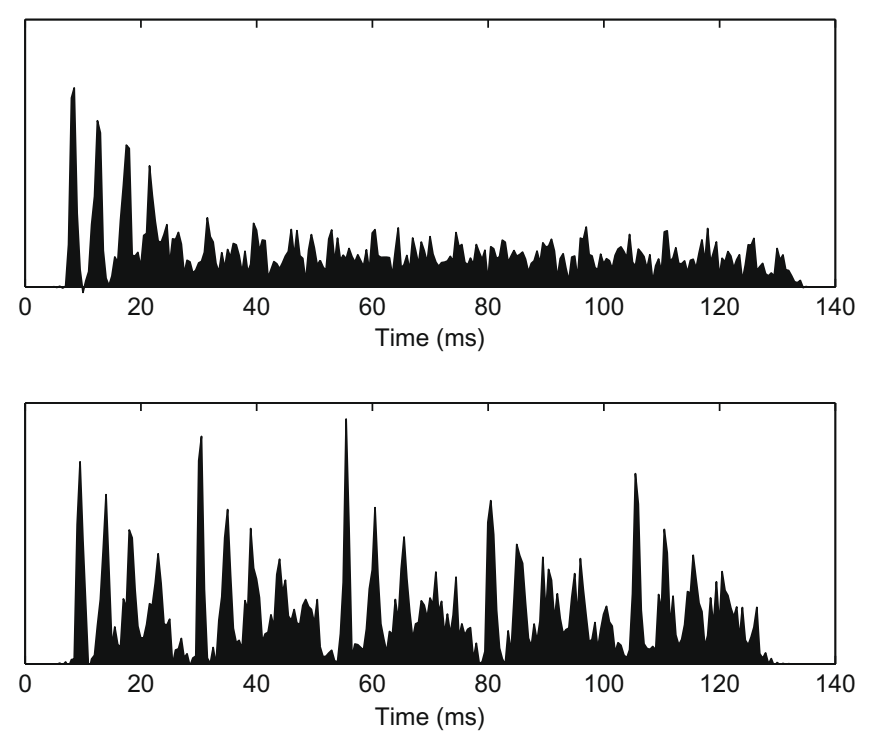

Fig. 3. Post-stimulus time histogram (PSTH) for a simulated T-stellate (chopper) neuron in response to: (a) a sinusoidal tone of $60 \mathrm{~dB}$ at $1 \mathrm{kHz}$ and duration of $250 \mathrm{~ms}$; (b) the same tone sinusoidally modulated at $100 \%$ depth with a modulation frequency of $40 \mathrm{~Hz}$.

Table 3

Network parameters values for the cochlear nucleus.

\begin{tabular}{lll}
\hline Parameter & Typical range (nS) & Description \\
\hline $\begin{array}{l}\text { Synaptic strengths } \\
g_{T T}\end{array}$ & $5-10$ & $\begin{array}{l}\text { Synaptic conductance } \\
\text { between T-stellate cells } \\
\text { Synaptic conductance } \\
\text { between D and T-stellate cells } \\
g_{D T}\end{array}$ \\
$g_{V T}$ & $5-25$ & $\begin{array}{l}\text { Synaptic conductance } \\
\text { between vertical and T-stellate cells } \\
\text { Synaptic conductance } \\
\text { between vertical and D-stellate cells } \\
\text { Synaptic conductance } \\
\text { between D-stellate and vertical cells }\end{array}$ \\
$g_{V D}$ & $5-25$ & \\
$g_{D V}$ & 15 &
\end{tabular}

ulation frequency of $40 \mathrm{~Hz}$. There is a clear reinforcing in the regularity of the discharge but, most interestingly, now the discharge is synchronized with the modulation frequency, even when the modulation period is not directly related to the initial rate of discharge $(250 \mathrm{~Hz})$. As we will see, this happens for a limited range of modulation frequencies only.

The onset-chopper behavior for the D-stellate cell in our model is obtained by means of the narrowband inhibitory action of the vertical cell projections. Removing this inhibition actually reinforces the activity after the onset spikes. This is most interesting because there is a long standing debate regarding whether the onset behavior could be a consequence of an internal mechanism of spike inhibition (Kalluri and Delgutte, 2003) or an outcome of the network and the inhibitory action of neighboring cells (Paolini and Clark, 1999). The D-stellate neurons respond more vigorously to noise because they receive wideband excitation but only narrowband inhibition. For a pure tone stimulus the delayed inhibition of the vertical cells (note that there is an explicit 2 ms delay in the synapse) almost suppresses the subsidiary spikes after the onset.

From the examination of the PSTH of stellate neurons, we determined a set of parameters that yields responses that were consistent with experimental observations. These values are given in Table 3.

\subsection{Modulation transfer functions}

Now we move to the study of time and rate modulation transfer functions of T-stellate and D-stellate neurons. These cells exhibit the most interesting responses since they are, for moderately high intensities, temporally tuned for a range of modulation frequencies, as reflected in their band-pass tMTF (Frisina et al., 1990a; Rhode and Greenberg, 1994). The modulation frequency causing the strongest synchronization is called the best modulation frequency (BMF). There is not a single value for BMFs, but a wide range of frequencies Rhode and Greenberg (1994).

In Fig. 4 we display the tMTF of the auditory nerve fibers for a modulated $1 \mathrm{kHz}$ tone at $50 \mathrm{~dB}$, as a reference. The fibers belong to the channel corresponding to the $\mathrm{CF}$ of the carrier. It is known that these tMTF are low-pass (Joris and Yin, 1992) for low intensity values and degrade for moderate and high intensities, as the fibers become saturated. Comparing to cutoff frequencies of AN fibers obtained by Joris and Yin (1992) it can be seen that our data are close to the $60 \mathrm{~Hz}$ value for their low-CF fibers.

We thus examined the tMTF of T-stellate cells using the set of parameters obtained in the previous section (see Table 3 ), and found a clear band-pass behavior with a BMF around $70 \mathrm{~Hz}$ (see Fig. 5, filled squares). This is a value somewhat low compared with the range $150-400 \mathrm{~Hz}$ given in (Rhode and Greenberg, 1994), but these authors were studying $C F>7 \mathrm{kHz}$ and observed a slightly decrease in the $B M F$ with the $C F$ (see Figs. 5 and 6 in last reference). Also we observed an upward shift of the peak for increasing values of intensity, in agreement with previously reported observations (Frisina et al., 1990a,b; Frisina, 2001; Frisina et al., 1997; Rhode and Greenberg, 1994). Comparing with Fig. 4, a striking enhancement of synchrony can be noticed, particularly for modulation frequencies greater than $60 \mathrm{~Hz}$.

Starting from the parameter values listed in Table 3, we explored the effect of the inhibition on the tMTF in our model. We found that when we increase the amount of inhibition of the vertical cell projections in the T-stellate units (controlled by $g_{V T}$ ) the peak raises and shifts towards lower modulation frequency values. The value of $g_{V T}$ was varied between 5 and $25 \mathrm{nS}$ shifting the BMF from 70 to $40 \mathrm{~Hz}$. Higher values of inhibition lead to degraded PSTH, are somewhat unrealistic and do not lead to a significative reduction of BMF. SI values are in the range 0.45-0.5, which are comparable to what was found in Rhode and Greenberg (1994) for $50 \mathrm{~dB}$ (we are using a $60 \mathrm{~dB}$ stimulus).

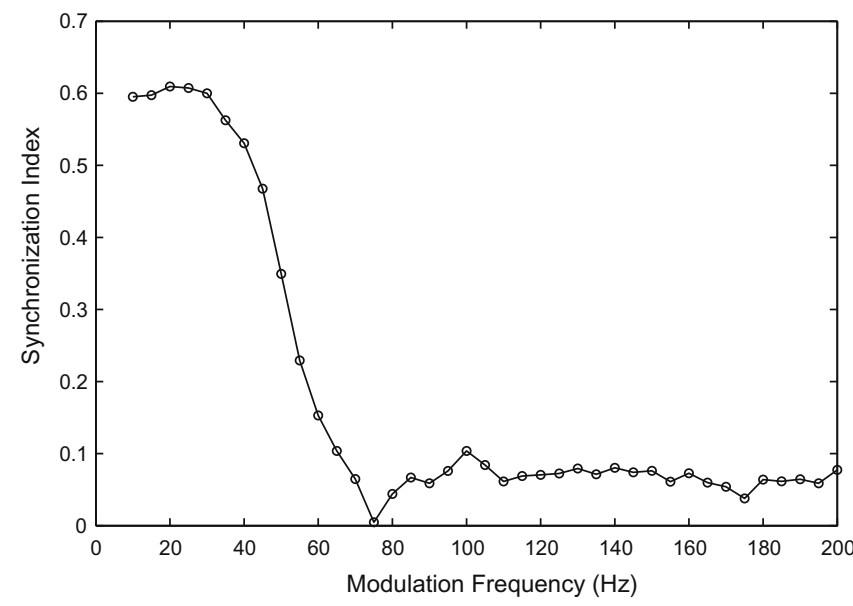

Fig. 4. Temporal modulation transfer function of the simulated auditory nerve fiber at the $\mathrm{CF}$, in response to $100 \% \mathrm{AM}$ stimuli, presented at $50 \mathrm{~dB}$ SPL, with carrier frequency $1 \mathrm{kHz}$ and modulation frequency from 10 to $200 \mathrm{~Hz}$, in $5 \mathrm{~Hz}$ steps. 


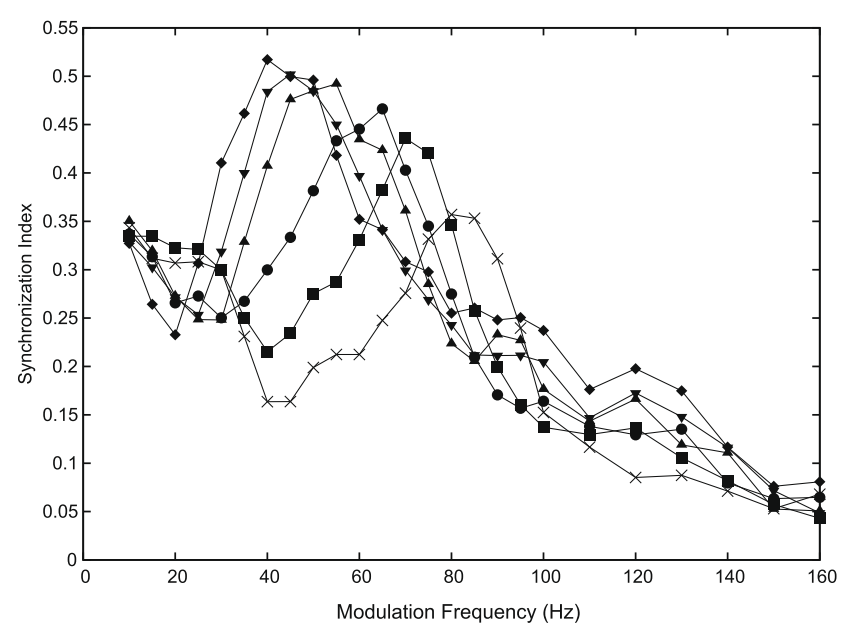

Fig. 5. Temporal modulation transfer functions for a simulated T-stellate neuron (chopper). Parameter values are given in the tables. Stimulus was a $500 \mathrm{~ms}$ tone, sinusoidally modulated in amplitude, at $60 \mathrm{~dB}$, presented 100 times for each of the modulation frequencies. Different curves correspond to different amounts of vertical cell inhibition: $g_{V T}=5 \mathrm{nS}$ (squares), $g_{V T}=10 \mathrm{nS}$ (circles), $g_{V T}=15 \mathrm{nS}$ (triangles up), $g_{V T}=20 \mathrm{nS}$ (triangles down), $g_{V T}=25 \mathrm{nS}$ (diamonds). We also show the results for the case of no inhibition $g_{V T}=0 \mathrm{nS}$ (crosses).

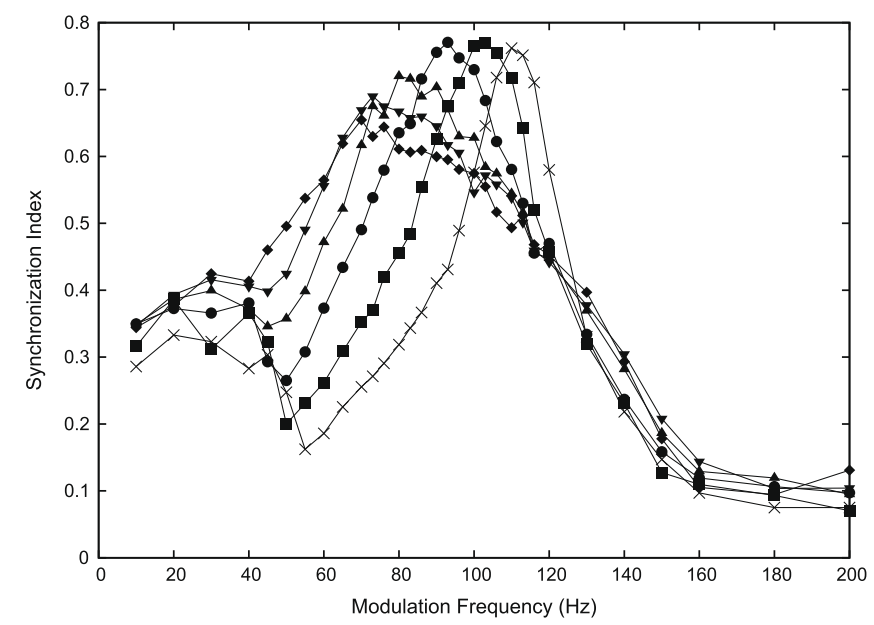

Fig. 6. Temporal modulation transfer functions for a simulated D-stellate neuron (onset-chopper). Stimulus was a $500 \mathrm{~ms}$ tone, sinusoidally modulated in amplitude, at $60 \mathrm{~dB}$, presented 100 times for each of the modulation frequencies. Different curves correspond to different amounts of vertical cell inhibition: $g_{V D}=5 \mathrm{nS}$ (squares), $g_{V D}=10 \mathrm{nS}$ (circles), $g_{V D}=15 \mathrm{nS}$ (triangles up), $g_{V D}=20 \mathrm{nS}$ (triangles down), $g_{V D}=25 \mathrm{nS}$ (diamonds). We also show the results for the case of no inhibition $g_{V D}=0 \mathrm{nS}$ (crosses).

Conversely, reducing inhibition increases the BFM and flatten the peak. In Fig. 5 we display a tMTF curve for $g_{V T}=0$, where there is still a band-pass at $80 \mathrm{~Hz}$ but with less synchronization than for the low modulation frequencies. Therefore we have a width of less than one octave for the BMF. The reported values of BMF span more than two octaves, but we will see that onset-chopper units could assist as a complementary source of BMF tuning.

This is a significant result, because the adjustable BFM could be the basis for a periodotopic organization in the cochlear nucleus. Moreover, the variation in the amount of inhibition modifies the modulation frequency tuning, without affecting the overall shape of the PSTH.

The rate modulation transfer function (rMTF) reflects how the rate of discharge depends on the modulation frequency, and is flat, for our model and in previously reported experimental results (Fri- sina et al., 1990a; Gai and Carney, 2008). As the parameter value controlling narrowband inhibition $\left(g_{V T}\right)$ is increased the mean discharge rate also increases in a linear way.

Previous studies (Rhode and Greenberg, 1994; Frisina et al., 1990a) have classified tMTF found in onset-chopper units as both low-pass and band-pass. We found, in our D-stellate units, a more conspicuous appearance of the band-pass behavior for a wide range of intensities and network parameters. This can be explained because the onset-chopper behavior is obtained with a great amount of vertical cell inhibition. As soon as this parameter is varied, the activity of the D-stellate cell increases after the onset discharge, displaying an irregular chopping behavior. This, probably unrealistic, chopping action of the D-stellate cells keeps the frequency selectivity in the tMFT.

We performed a parameter variation similar to that done on $\mathrm{T}$ Stellate cells, but now increasing and decreasing the amount of inhibition on the D-stellate cells only. Hence, we varied $g_{V D}$ in the range 5-25 nS. The tMTF obtained for D-stellate cells for the different values of $g_{V D}$ is displayed in Fig. 6. We also show a curve for $g_{V D}=0$. As before, increasing the amount of inhibition decreases the value of the BMF, and in this case the tuning covers the range $70-110 \mathrm{~Hz}$. The values of synchronization at BMF are higher than those for choppers (between 0.65 and 0.8 ), a feature that is characteristic for onset-choppers.

In contrast to chopper units, the PSTH of the onset-chopper cells is harshly degraded when the amount of DCN inhibition is varied. The weakness of the D-stellate PSTH can be explained since we are not taking into account all the possible sources of inhibition. For example, experimentally demonstrated GABAergic inhibition coming from granule cells (Ferragamo et al., 1998).

However, since there are some D-stellate units with band-pass tMTF, and we observed that it is feasible the inhibition-based tuning mechanism, it could be possible that some of those cells can be also part of a periodotopic organization in the cochlear nucleus.

\section{Discussion}

For the development of an accurate model of the $\mathrm{CN}$, it is crucial to have a well timed inputting auditory nerve spike train, with proper statistics and dynamical range. For this reason, we implemented a detailed biophysical model of the periphery, that we believe has the advantage of possessing parameters that correspond to physiologically meaningful measurable quantities. These cannot only be contrasted with experiments when available, but adapted in order to represent different species of mammals. Model parameters for which no experimental records were available were fitted within the limits imposed by biological results obtained in other cellular or neural systems. In this spirit, a detailed calcium dynamics at ribbon synapses was introduced, allowing a more realistic simulation of the dynamical range and specific spontaneous discharge rates of the different auditory nerve fiber types.

On the other hand, most models of the auditory periphery present in the literature are phenomenological. The shortcoming is that they are adjusted for a certain range of stimulus parameters, both in intensity and spectral distribution. To overcome this, we tried to achieve an adjustment similar to those observed in previous models, but also to expand the range of applicability while maintaining a comparable correlation with actual biological systems. Furthermore, we believe that another important point concerning the codification of complex sounds regards the relative timing between the different basilar membrane channels. In opposition with filter-bank models of the cochlea, the present model carries this channel information naturally.

When representing the cochlear nucleus the approach was quite different. Since the $\mathrm{CN}$ presents an important variety of neu- 
ral populations and a relatively complex neuropil, and keeping in mind that we are more interested in representing processes than the complete picture of the CN physiological "zoo", we opted to implement this region with the highest degree of simplicity that still allowed the system to perform the task. Importantly, neural network connections and most of the parameters used had been experimentally examined by previous studies, imposing a great biological constraint to the dynamics of the model.

In an overall view, although the results presented do not span the complete dynamical picture of the model due to the restricted survey of the parameter space, the results obtained satisfactorily replicated the general behavior of the biological actors involved, without imposing artificial constraints. Importantly, the model was able to reproduce a consistently observed feature of the auditory system: an enhancement in AM information coding in the $\mathrm{CN}$ with respect to the AN. Fibers of the AN show a flat rMTF and some degree of untuned or unselective synchronization reflected in a low-pass tMTF. Depending on signal intensity, neurons at the $\mathrm{CN}$, specially chopper ones, evidence a significative selective temporal synchronization (i.e., "bell-shaped" tMTFs) and no selectivity in their rate response. These characteristics were found in our model too.

Another important observation that can be pointed out from the presented analysis, concerns the neural coding mechanism of AM sounds at central levels. A topic of debate involves the hypothesis of the existence of so-called "modulation frequency filter banks" (Kay and Matthews, 1972). These are no more than band-pass filters centered at different modulation frequencies (BMFs) that would yield specific neural populations sensitive to narrow bands of modulation frequencies, resulting in a population encoding of AM information. This suggestion is supported by the above mentioned experimental observation of $\mathrm{CN}$ chopper neurons possessing band-pass tMTFs, tuned to different BMFs, and therefore sensitive to phase-lock their response patterns to particular narrow bands of modulation frequencies, while keeping their rate response relatively constant. Interestingly, there have been claims that, at the level of the inferior colliculus, these filter banks are topographically organized (Schreiner and Langner, 1988). Any model of these processes needs to explain the neural mechanism responsible for the tuning of neurons to different modulation frequency bands. The results of this simple model suggest that it is possible to shift the peak of band-pass tMTFs to different modulation frequencies just by adjusting the level of inhibition imposed by glycinergic vertical neurons, being that higher levels of inhibition resulted in lower temporal BMFs. For the relatively short range of parameters tested, exciting with a carrier frequency of $1 \mathrm{kHz}$, resulted in a shift corresponding to one octave, in the approximate $60-110$ and $30-80 \mathrm{~Hz}$ range for onset and chopper neurons respectively, which is a significant proportion of the carrier frequency. A much wider range could be hardly expected, because there is some evidence that the selective responses to low modulation frequencies are predominantly generated at the cortical level, at least in humans (John and Picton, 2000), and higher modulation frequency synchrony is limited by the proximity of the characteristic frequency of $1 \mathrm{kHz}$. It remains to be explored how this frequency range changes as the $\mathrm{CF}$ is increased. It also remains to be studied if different input signals and further parameter adjustments result in a wider dynamical range. However, the observed principle of inhibition force acting on chopper and onset cells being inversely proportional to their temporal BMFs is quite robust, and biologically significant, since the scenario of $\mathrm{CN}$ neurons receiving a spectrum of different inhibition forces stands as a physiologically plausible mechanism for the tuning of tMTFs. In fact, it has been shown that the glycine antagonist strychnine is capable of producing a significative modulation of the AM synchronization of onset and chopper neurons (Frisina et al., 1997; Gai and Carney, 2008), underpinning our observed results.

Previous works modeled VCN neurons response to AM stimuli. An approach that has been taken to calibrate the neural tMTFs to different BMFs was the adjustment of membrane and potassium conductance time constants (Hewitt and Meddis, 1994; Guérin et al., 2006). For example, using membrane time constants in the order of $1 \mathrm{~ms}$ and conductance time constants in the $0.1-10 \mathrm{~ms}$ range for point neuron models of VCN chopper neurons, results in BMFs in the $40-400 \mathrm{~Hz}$ range (Hewitt and Meddis, 1994). Besides the fact that tuning neuronal BMFs through ad-hoc calibration of conductance time constants can be regarded as a biologically Demanding assumption (specially when one considers the lack of calibration freedom that these parameters present, since they are molecularly determined), using a 100 -fold range of time constants for a unique and somewhat physiologically homogeneous population of neurons seems biologically unrealistic. The BMF tuning by means of inhibition force modulation presented in this work stands, in our opinion, as a more plausible alternative. Moreover, the limited set of simulations developed in this study was able to produce a significative calibration of BMFs using a much more restricted dynamic range of the tuning parameter (a 5 -fold variation in inhibition).

In conclusion, the present work is a simple theoretical approach to the understanding of AM signal processing in the first steps of auditory system. Through the development of a realistic biophysical model of the periphery and a more simplified neural network model of the $\mathrm{CN}$, we were able to reproduce several behaviors observed in experimental studies. Some of these where mimetic of important aspects of neural AM encoding, such as the enhancement of AM information carried in the activity patterns of $\mathrm{CN}$ neurons with respect to the auditory nerve and the generation of $\mathrm{CN}$ neurons tMTFs tuned to phase-lock to a restricted, yet significative, range different BMFs. Importantly, no artificial constraints were introduced to produce this tuning, since it was the consequence of the degree of inhibition suffered by these cells. Further analysis of the model's parameter space should be carried out for a better comprehension and validation of its dynamics and in order to expand the scope of the results.

\section{Acknowledgments}

This research was supported by Agencia de Promoción Científica y Tecnológica (FONCyT - PICT 32391) and CONICET. SAR receive support from Conselho Nacional de Desenvolvimento Científico e Tecnológico (CNPq).

\section{References}

Arnott, R.H., Wallace, M.N., Shackleton, T.M., Palmer, A.R., 2004. Onset neurones in the anteroventral cochlear nucleus project to the dorsal cochlear nucleus. JARO $5,153-170$

Bahmer, A., Langner, G., 2006. Oscillating neurons in the cochlear nucleus: I. Experimental basis of a simulation paradigm. Biol. Cybern. 95, 371-379.

Bahmer, A., Langner, G., 2006. Oscillating neurons in the cochlear nucleus: II. Simulation results. Biol. Cybern. 95, 381-392.

Borst, M., Knoblauch, A., Palm, G., 2004. Modelling the auditory system: preprocessing and associative memories using spiking neurons. Neurocomputing 58, 1013-1018.

Carney, L.H., 1993. A model for the responses of low-frequency auditory-nerve fibers in cat. J. Acoust. Am. 93 (1), 401-417.

Choe, Y., Magnasco, M.O., Hudspeth, A.J., 1998. A model for amplification of hair cell-bundle motion by cyclical binding of $\mathrm{Ca}^{2+}$ to mechanical-transduction channel. Proc. Natl. Acad. Sci. USA 95, 15321-15326.

Dallos, P., 1996. Overview: cochlear neurobiology. In: Dallos, P., Popper, A.N., Fray, R.R. (Eds.), The Cochlea. Springer, New York, NY, pp. 1-43.

Destexhe, A., Mainen, Z.F., Sejnowski, T.J., 1999. Kinetic models of synaptic transmission. In: Koch, C., Segev, I. (Eds.), Methods in Neuronal Modelling, second ed. MIT Press, Cambridge, MA, pp. 1-26. 
Dicke, U., Ewert, S.D., Dau, T., Kollmeier, B., 2006. A neural circuit transforming temporal periodicity information into a rate-based representation in the mammalian auditory system. J. Acoust. Soc. Am. 121, 310-326.

Doucet, J.R., Ryugo, D.K., 2006. Structural and functional classes of multipolar cells in the ventral cochlear nucleus. Anat. Rec., Part A 288A, 331-344.

Doucet, J.R., Ross, A.T., Gillespie, M.B., Ryugo, D.K., 1999. Glycine immunoreactivity of multipolar neurons in the ventral cochlear nucleus which project to the dorsal cochlear nucleus. J. Comp. Neurol. 408, 515-531.

Eggermont, J.J., 2001. Between sound and perception: reviewing the search for a neural code. Hear. Res. 157, 1-42.

Eriksson, J.L., Robert, A., 1999. The representation of pure tones and noise in a model of cochlear nucleus neurons. J. Acoust. Soc. Am. 106, 1865-1879.

Fassel, R., Kohlrausch, A., 1995. Modulation detection as a function of carrier frequency and level. IPO Annu. Prog. Rep. 30, 21-29.

Fastl, H., Zwicker, E., 2007. Psychoacoustics, fifth ed. Springer, Berlin.

Ferragamo, M.J., Golding, N.L., Oertel, D., 1998. Synaptic inputs to stellate cells in the ventral cochlear nucleus. J. Neurophysiol. 79, 51-63.

Frisina, R.D., 2001. subcortical neural coding mechanisms for auditory temporal processing. Hear. Res. 158, 1-27.

Frisina, R.D., Smith, R.L., Chamberlain, S.C., 1990a. Encoding of amplitude modulation in the gerbil cochlear nucleus: I. A hierarchy of enhancement. Hear. Res. 44, 99-122.

Frisina, R.D., Smith, R.L., Chamberlain, S.C., 1990b. Encoding of amplitude modulation in the gerbil cochlear nucleus: II. Possible neural mechanisms. Hear. Res. 44, 123-142.

Frisina, R.D., Wang, J., Byrd, J.D., Karchich, K.J., Salvi, R.J., 1997. Enhanced processing of temporal features of sound in background noise by cochlear nucleus single neurons. In: Skya, J. (Ed.), Acoustical Signal Processing in the Central Auditory System. Plenum Press, New York, NY, pp. 109-125.

Gai, Y., Carney, L.H., 2008. Influence of inhibitory inputs on rate and timing of responses in the anteroventral cochlear nucleus. J. Neurophysiol. 99, 10771095.

Gardner, S.M., Trusell, L.O., Oertel, D., 1999. Time course and permeation of synaptic AMPA receptors in cochlear nucleus correlate with input. J. Neurosci. 19, 87218729.

Goutman, J.D., Glowatzki, E., 2007. Time course and calcium dependence of transmitter release at a single ribbon synapse. PNAS 104, 16341-16346.

Guérin, A., Le Bouquin Jeannès, R., Bès, J., Faucon, G., Lorenzi, C., 2006. Evaluation of two computational models of amplitude modulation coding in the inferior colliculus. Hear. Res. 211, 5462.

Hewitt, M.J., Meddis, R., 1994. A computer model of amplitude-modulation sensitivity of single units in the inferior colliculus. J. Acoust. Soc. Am. 95, 2145-2159.

Hewitt, M.J., Meddis, R., Shackleton, T.M., 1991. A computer model of a cochlearnucleus stellate cell: responses to amplitude-modulated and pure-tone stimuli. J. Acous. Soc. Am. 91, 2096-2109.

Hudspeth, A.J., Lewis, R.S., 1988. Kinetic analysis of voltage and ion dependent conductances in saccular hair cells of the bull-frog, Rana catesbeiana. J. Physiol. 400, 237-274.

John, M.S., Picton, T.W., 2000. Human auditory steady-state responses to amplitudemodulated tones: phase and latency measurements. Hear. Res. 141, 57-79.

Johnson, D.H., 1980. The relationship between spike rate and synchrony in responses of auditory-nerve fibers to single tones. J. Acoust. Soc. Am. 68, $1115-1122$.

Joris, P.X., Yin, T.C.T., 1992. Responses to amplitude-modulated tones in the auditory nerve of the cat. J. Acoust. Soc. Am. 91, 215-232.

Joris, P.X., Shreiner, C.E., Rees, A., 2004. Neural processing of amplitude-modulated sounds. Physiol. Rev. 84, 541-577.

Kalluri, S., Delgutte, B., 2003. Mathematical models of cochlear nucleus onset neurons: II. Model with dynamic spike-blocking state. J. Comput. Neurosci. 14, 91110

Kay, R.H., Matthews, D.R., 1972. On the existence in human auditory pathways of channels selectively tuned to the modulation present in frequency-modulated tones. J. Physiol. 225, 657677.

Krishna, B.S., Semple, M.N., 2000. Auditory temporal processing: responses to sinusoidally amplitude-modulated tones in the inferior colliculus. J. Neurophisiol. 84, 255-273.

Langner, G., 1992. Periodicity coding in the auditory system. Hear. Res. 60, 115142.
Liberman, M.C., 1978. Auditory-nerve responses from cats raised in a low-noise chamber. J. Acoust. Soc. Am. 63, 442-455.

Lopez Poveda, E., Martin, A.E., 2006. A biophysical model of the inner hair cell: the contribution of potassium currents to peripheral auditory compression. JARO 7, 218-235.

Meddis, R., 1986. Simulation of mechanical transduction in the auditory receptor. J. Acoust. Soc. Am. 79, 702-711.

Møller, A., 1974. Responses of units in the cochlear nucleus to sinusoidally amplitude modulated tones. Exp. Neurol. 45, 104-117.

Moser, T., Brandt, A., Lysakowski, A., 2006. Hair cell ribbon synapses. Cell Tissue Res. 326, 347-359 (J. Acoust .Soc. Am. 95 (4), 2145-2149).

Needham, K., Paolini, A.G., 2006. Neural timing, inhibition and the nature of stellate cell interaction in the ventral cochlear nucleus. Hear. Res., 31-42.

Nelson, P.C., Carney, L.H., 2004. A phenomenological model of peripheral and central neural responses to amplitude-modulated tones. J. Acoust. Soc. Am. 116, 2173-2186.

Nobili, R., Mammano, F., Ashmore, J., 1998. How well do we understand the cochlea? TINS 21 (4), 159-167.

Nobili, R., Vetesnik, A., Turicchia, L., Mammano, F., 2003. Otoacustic emissions from residual oscillations of the cochlear basilar membrane in human ear model. JARO 4, 478-494.

Osen, K.K., 1969. Cytoarchitecture of the cochlear nuclei in the cat. J. Comp. Neurol. $136,453-483$.

Palmer, A.R., Wallace, M.N., Arnott, R.H., Shackleton, T.M., 2003. Morphology of physiologically characterised ventral cochlear nucleus stellate cells. Exp. Brain Res. 153, 418-426.

Paolini, A.G., Clark, G.M., 1999. Intracellular responses of onset chopper neurons in the ventral cochlear nucleus to tones: evidence for dual-component processing. J. Neurophysiol. 81, 2347-2359.

Paolini, A.G., Clarey, J.C. Needham, K. Clark, G.M., 2005. Balanced inhibition and excitation underlies spike firing regularity in ventral cochlear nucleus chopper neurons. Eur. J. Neurosci. 21, 1236-1248.

Pressnitzer, D., Meddis, R., Delahaye, R., Winter, I.M., 2001. J. Neurosci. 21, 63776386.

Rhode, W.S., 1999. Vertical cell responses to sound in cat dorsal cochlear nucleus. J. Neurophysiol. 82, 1019-1032.

Rhode, W.S., Greenberg, S., 1994. Encoding of amplitude modulation in the cochlear nucleus of the cat. J. Neurophysiol. 71, 1797-1925.

Rodríguez-Contreras, A., Yamoah, E.N., 2003. Effects of permeant ion concentrations on the gating of L-type $\mathrm{Ca}^{2+}$ channels in hair cells. Biophys. J. 84, 3457-3469.

Rose, J., Galambos, R., Hughes J., 1960. Organization of frequency sensitive neurons in the cochlear nuclear complex of the cat. In: Rasmussen, G., Windle, W. (Eds.), Neural Mechanisms of the Auditory and Vestibular Systems. Thomas Springfield, pp. 116-136.

Rothman, J.S., Manis, P.B., 2003. The roles potassium currents play in regulating the electrical activity of ventral cochlear nucleus neurons. J. Neurophysiol. 89, 3097-3113.

Ryugo, D.K., Parks, T.N., 2003. Primary innervation of the avian and mammalian cochlear nucleus. Brain Res. Bull. 60, 435-456.

Schreiner, C.E., Langner, G., 1988. Periodicity coding in the inferior colliculus of the cat. II. Topographical organization. J. Neurophysiol. 60, 1823-1840.

Shamma, S.A., Chadwwick, R.S., Wilburn, W.J., Morrish, K.A., Rinzel, J., 1986. A biophysical model of cochlear processing: intensity dependence of pure tone response. J. Acoust. Soc. Am. 80, 43-63.

Sikora, M.A., Gottesman, J., Miller, R.F., 2005. A computational model of the ribbon synapse. J. Neurosci. Methods 145, 47-61.

Sumner, C.J., Lopez Poveda, E.A., OMard, L.P., Meddis, R., 2002. A revised model of the inner hair cell and auditory-nerve complex. J. Acoust. Soc. Am. 111 (5), 2178-2188.

Wickesberg, R.E., Oertel, D., 1991. Tuberculoventral neurons project to the multipolar cell area but not to the octopus area of the posteroventral cochlear nucleus. J. Comp. Neurol. 15, 457-468.

Young, E.D., Oertel, D., 2004. Cochlear nucleus. In: Shepherd, G.M. (Ed.), The Synaptic Organization of the Brain, fifth ed. Oxford University Press, New York, NY, pp. 125-163.

Zhang, X., Carney, L.H., 2005. Analysis of models for the synapse between the inner hair cell and the auditory nerve. J. Acoust. Soc. Am. 118 (3), 1540-1553.

Zhang, S., Oertel, D., 1993. Tuberculoventral cells of the dorsal cochlear nucleus of mice: intracellular recordings in slices. J. Neurophysiol. 69, 1409-1421. 